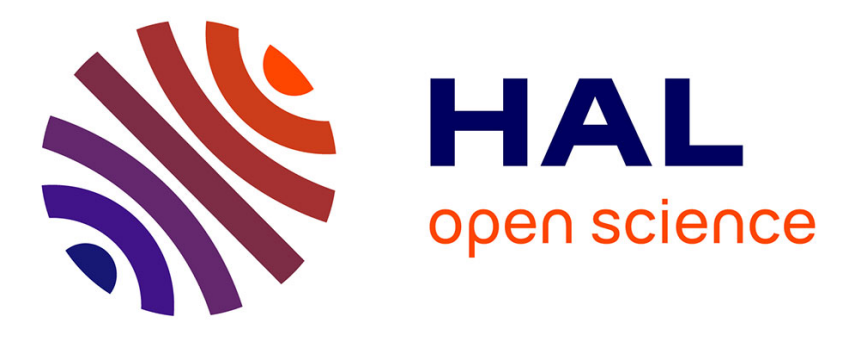

\title{
On the design of a preshaped curved beam bistable mechanism
}

\author{
Hussein Hussein, Patrice Le Moal, Rafic Younes, Gilles Bourbon, Yassine \\ Haddab, Philippe Lutz
}

\section{- To cite this version:}

Hussein Hussein, Patrice Le Moal, Rafic Younes, Gilles Bourbon, Yassine Haddab, et al.. On the design of a preshaped curved beam bistable mechanism. Mechanism and Machine Theory, 2019, 131, pp.204-217. 10.1016/j.mechmachtheory.2018.09.024 . lirmm-02006749

\section{HAL Id: lirmm-02006749 \\ https://hal-lirmm.ccsd.cnrs.fr/lirmm-02006749}

Submitted on 4 Feb 2019

HAL is a multi-disciplinary open access archive for the deposit and dissemination of scientific research documents, whether they are published or not. The documents may come from teaching and research institutions in France or abroad, or from public or private research centers.
L'archive ouverte pluridisciplinaire HAL, est destinée au dépôt et à la diffusion de documents scientifiques de niveau recherche, publiés ou non, émanant des établissements d'enseignement et de recherche français ou étrangers, des laboratoires publics ou privés. 


\title{
On the design of a preshaped curved beam bistable mechanism
}

\author{
Hussein Hussein $^{\mathrm{a}}$, Patrice Le Moal ${ }^{\mathrm{b}}$, Rafic Younes $^{\mathrm{c}}$, Gilles Bourbon ${ }^{\mathrm{b}}$, \\ Yassine Haddab ${ }^{\mathrm{a}}$, Philippe Lutz ${ }^{\mathrm{b}}$ \\ ${ }^{a}$ LIRMM, Université de Montpellier, CNRS, Montpellier, France \\ ${ }^{b}$ Femto-st, Université de Franche Comté, CNRS, Besancon, France \\ ${ }^{c}$ Lebanese university, Faculty of engineering, Hadath, Lebanon
}

\begin{abstract}
The preshaped curved beam is used as bistable mechanism in MEMS, its behavior is characterized by the snapping forces and stresses that evolve during deflection between the two sides of buckling. Based on analytical models, the influence of variating the material and dimension parameters on the behavior of the curved beam is analyzed. The limit of miniaturization of the beam based on the stress limits is identified. Further, a design optimization methodology is provided which allows selecting the miniature dimensions of the curved beam after defining the range of possible dimensions. This method ensures a required bistablity behavior in terms of stroke and holding forces, while respecting the design requirement. Several design constraints are defined including material and technological limitations and some other specifications. The results in different cases of optimization problems showed to be the same using a proposed optimization methodology and a standard gradient based optimization algorithm.
\end{abstract}

Keywords: Preshaped curved beam, Design methodology, Miniaturization

\section{Introduction}

The design of a preshaped curved beam is still paying the price for the lack of a physical intuition and a methodology of optimization. The design of a preshaped curved beam is investigated in this paper in an effort to provide 5 efficient computational frameworks and elements for designers.

Curved beam structure is a component widely used in MEMS as a simple bistable mechanism that combines the advantages of passive holding and compliant mechanisms. The bistable structure is generally simple which allows working easily in confined spaces. The stable positions are robust and have 10 good repeatability while no energy is required to hold the positions. Compared

\footnotetext{
${ }^{*}$ Corresponding author

Email address: hussein.hussein@lirmm.fr (Hussein Hussein)
} 
to precompressed [1, 2, 3] and prestressed [4, 5] curved beams, the preshaped curved beam has an advantage at microfabrication level insofar as the integration in MEMS is simpler and monolithic microfabrication is possible [6, 7, 8, 9]. All of those are advantages, as far as the miniaturization of mechatronic systems 15 is concerned.

The analytical modeling constitutes a basis for the design of these kinds of beams. Several studies in the literature covered the analytical modeling of preshaped curved beams [7, 8, 9, 10, 11, 12, 13, 14, 15]. These models are either static or dynamic. However, the dynamic behavior of a curved beam is very 20 fast compared to its most common applications in MEMS devices (switch [16], positioning [17, relays [18, braille devices [19], etc.). The design study in this paper aims to optimize the quasi-static behavior of the curved beam. For other fast applications (such as resonators 20]), where the dynamic is important, the design can be relied on dynamic modeling [13, 14, 15.

25 As for the static modeling, the bistability and pull-in behavior of electrostatically actuated preshaped curved beam was studied in [10, 11, 21]. The modeling of electromagnetically actuated preshaped curved beams is presented in [7. The modeling of a preshaped curved beam actuated by a point force at its middle length was investigated in [8] with neglecting high modes of buckling. Expres30 sions describing the snapping force behavior during deflection between the two sides of buckling were obtained and showed good accordance with FEM simulations and experiments. The modeling with considering high modes of buckling was presented recently in [9]. Expressions for the snapping forces and internal stresses were developed where considering high modes of buckling showed better 35 accordance with FEM results, mainly for the stress calculation. The snapping forces calculated from [8, 9] showed also good accordance with the experiments made in 22]. For the purpose of generalization, numerical modeling of an arbitrarily preshaped curved beam subjected to arbitrary distributed mechanical and/or electrostatic loadings is presented in [12]. To the best of the author's

40 knowledge, no existing research addresses the dimensioning and optimization of preshaped curved beams.

The analytical expressions obtained in the modeling constitute a basis for the design. The accurate determination of the relation between the design parameters of the curved beam (material and dimensions) and the snapping forces 45 is very important for the integration of the curved beam in a complete microsystem. At the same level, determination of the maximal stress value according to the material and dimensions allows the identification of the miniaturization limits and avoidance of the fracture.

The design of the preshaped curved beam is investigated in this paper based 50 on the analytical expressions provided in the modeling. In a first part, the influence of variating the material and dimension parameters on the main characteristics that define the behavior of the curved beam are investigated. These characteristics are the important snapping positions and forces, internal stress and limits of miniaturization. In a second part, a design optimization methodol-

${ }_{55}$ ogy is presented and developed to identify the miniature dimensions that allow an expected bistable behavior. Different design constraints including material 
and technological limitations and some other specification are presented and their corresponding conditions on the dimensioning are defined. Afterwards, the optimization problem is solved using standard gradient based optimization algorithm and a visual interpretation methodology. The optimization results using the two methods showed to be the same in different presented cases.

The works made in this paper seek to fill the gap between the modeling and the real dimensioning of preshaped curved beams. The elements provided constitute a basis for the design of a preshaped curved beam in order to get

65 its most miniature dimensions while providing a set of design requirements. Nevertheless, provided elements constitute a complete toolbox for designers. They can be changed easily to suit other design requirements and optimization scenarios. Further, knowing that MEMS costs are closely related to their surface footprints, the design elements presented in this paper constitute a basis for the economical study of the final realization of preshaped curved beam based systems.

\section{Model}

The model in question is a clamped-clamped preshaped curved beam which is fabricated directly on the shape of the first mode of buckling. This shape is most commonly reported 8, 9, 23, since it is similar to bistable buckled beam resulting from axially compressed or prestressed straight beam. Figure 1 shows a schematic of a preshaped curved beam with the different dimension parameters: depth $b$, thickness $t$, length $l$ and initial height of buckling $h$.

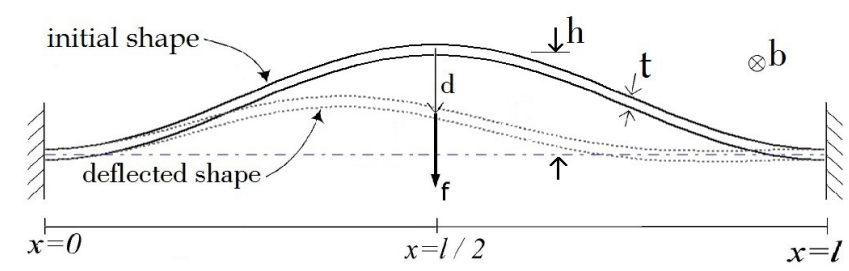

Figure 1: Schematic of a preshaped curved beam in its initial position and during deflection.

An applied lateral force $f$ at the middle of the curved beam causes a deflec-

80 tion $d$ at its middle as shown in Figure 1. A simple one preshaped curved beam shows bistability only for high values of the height-to-thickness ratio $Q=h / t$. The bistability, in this case, is highly asymmetric and the stability margin is very low in the second side of buckling.

Usually, a bistable mechanism of preshaped curved beams consists of two curved beams at least that are connected by a shuttle at the middle as shown in Figure 2,

The deflection in this case is constrained; the asymmetrical modes of buckling are eliminated and a rectilinear displacement of the shuttle is ensured during deflection. The bistability of a constrained preshaped curved beam is ensured for 


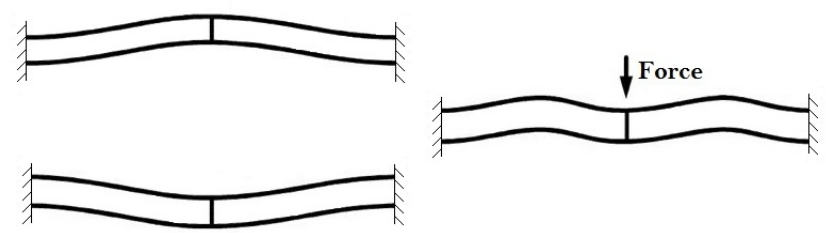

Figure 2: Pair of preshaped curved beams as bistable mechanism shown during deflection between the two sides of buckling.

90 $Q \geq 2.31$ [8, 9. The design study in this paper concerns constrained preshaped curved beams.

The snapping forces and internal stresses evolve in two different ways during deflection of the curved beam between the two sides of buckling. During deflection from one side, the length of the beam is compressed and the axial stress increases with deflection. Behavior of the force with the deflection is nonlinear in this zone. However, after a certain limit of deflection, particularly in an intermediate zone between the two sides of buckling, the axial stress becomes constant due to the appearance of the third mode of buckling in the shape of the curved beam. Behavior of the force becomes linear with the deflection in this zone.

Figure 3 shows characteristic curve of the snapping force during deflection. The zone between $d_{t o p}$ and $d_{b o t}$ is the linear zone, while the force behavior outside this zone is non linear.

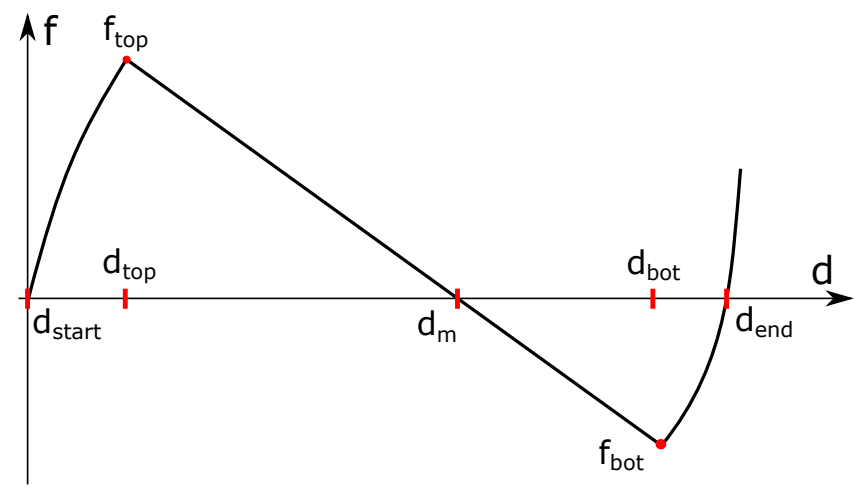

Figure 3: Snapping force characteristic curve during deflection of a preshaped curved beam.

For $Q>2.78$, the top point $\left(d_{\text {top }}, f_{\text {top }}\right)$ is the highest point on the curve, while for $Q>2.71$, the bottom point $\left(d_{b o t}, f_{b o t}\right)$ is the lowest point on the curve. For lower values of $Q$, the higher and lower force points are in the nonlinear zone. Usually, in a bistable curved beam, the ratio $Q$ is higher than 2.78 and the top and bottom points are the higher and lower points respectively.

The existence of positive and negative values of the snapping force indicates 
the existence of two stable positions in the two buckling sides. The stability margin in the second side of buckling increases for higher values of $Q$. In case of no external applied forces at rest, $d_{\text {start }}$ and $d_{\text {end }}$ are the two stable positions and the distance between them is the stroke of the curved beam.

Otherwise, the stroke can be defined differently using stop blocks [17] that limit the curved beam position between two stop positions in between $d_{\text {start }}$ and $d_{\text {end }}$. This allows defining the stroke between stable positions and equally the holding force at each position. The design method presented in section 4 is based on the use of stop blocks to define the stroke and holding forces.

The different snapping points presented in Figure 3 are dependent on the dimension and material parameters as can be concluded from their expressions [9]:

$$
\begin{aligned}
& \left\{d_{\text {start }}, d_{m}, d_{\text {end }}\right\}=h \cdot\left\{0, \frac{4}{3}, \frac{3}{2}+\sqrt{\frac{1}{4}-\frac{4}{3 Q^{2}}}\right\} \\
& d_{\text {top }}, d_{\text {bot }}=h \cdot\left(\frac{28}{27} \pm \frac{2 \pi}{3} \sqrt{\frac{1}{6}+\frac{16}{81 \pi^{2}}-\frac{1}{Q^{2}}}\right) \\
& f_{\text {top }}, f_{\text {bot }}=\frac{E b t^{3} h}{l^{3}} \cdot \frac{32 \pi^{2}}{9}\left(\frac{4}{9} \mp \pi \sqrt{\frac{1}{6}+\frac{16}{81 \pi^{2}}-\frac{1}{Q^{2}}}\right)
\end{aligned}
$$

where $E$ is the Young's modulus for the material. The existence of the above snapping points is not ensured for $Q<2.31$ (signs of expressions under the square root are negative) where the bistability condition is not satisfied.

The stress state in the curved beam consists of bending and axial stresses. As the case of the snapping force, the total stress, which is the sum of the bending and axial stresses, evolves in two ways between the two zones of deflection [9]. The most relevant stress value for the design is the value of the maximal stress along the curved beam during deflection $\sigma_{\text {max }}^{d}$. Figure 4 shows the curve shape of $\sigma_{\max }^{d}$ during deflection.

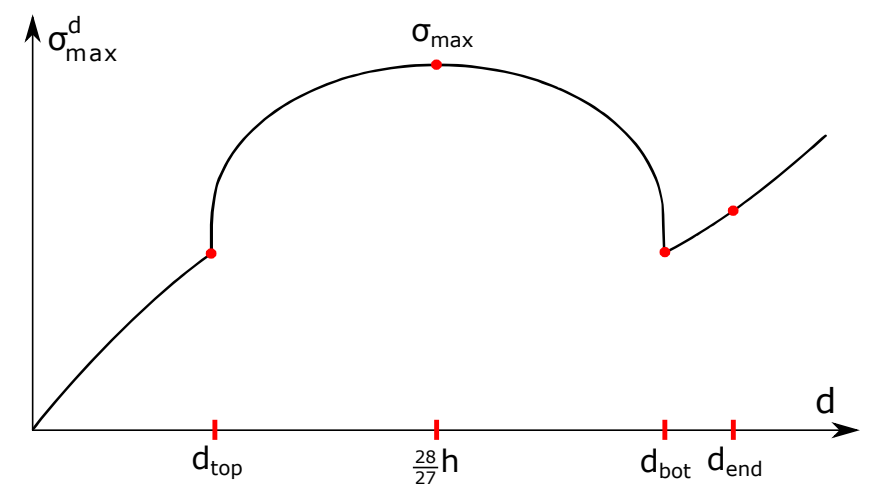

Figure 4: Curve shape of the maximal stress in a bistable preshaped curved beam during deflection.

130

The modeling shows that the maximum value $\sigma_{\max }$ of $\sigma_{\max }^{d}$ along the dis- 
tance between $d_{\text {start }}$ and $d_{\text {end }}$ is at $d=28 h / 27$ for $Q>2.36$. The expression of $\sigma_{\max }$ is as follows [9]:

$$
\sigma_{\text {max }}=\frac{E t h}{l^{2}} \frac{4 \pi^{2}}{3}\left(1+\frac{1}{Q}+\sqrt{\frac{64}{27 \pi^{2}}+2-\frac{12}{Q^{2}}}\right)
$$

\section{Parameters variation influence on the mechanical characteristics}

A key starting point in the design of a preshaped curved beam is to understand the influence of changing the different dimensions and material parameters on the mechanical characteristics of the curved beam. The relations between the different parameters and characteristics are analyzed in this section.

\subsection{Positions, forces and stresses}

As can be concluded from (1) and (2), the different positions, force and stress points are dependent directly or inversely on a power function of the material Young's modulus, the depth $b$ and the length $l$. However, the relation with the height $h$ and the thickness $t$ is more complicated since both are related to each other with respect to the ratio $Q$. Usually, the value of $Q$ is not a design requirement, but it strongly influences the behavior of the preshaped curved beam.

For constant values of $Q$, the height $h$ and the thickness $t$ evolve simultaneously with the same ratio. In this case, the position, force and stress characteristics are related directly to a power function of $h$ and $t$. However, changing $h$ and $t$ differently induces a variation on the value of $Q$ which has a nonlinear influence on the values of the different characteristics. In the other side, for high values of $Q$ (let's say $Q>6$ ), the nonlinear influence of variating $Q$ can be neglected. Therefore, the different characteristics can be considered as proportional to a power function of $h$ and $t$ for high values of $Q$.

In light of that, the different positions, force and stress points can be expressed in two ways as follows:

$$
\begin{aligned}
& d_{*}=t \cdot f_{1 h *}(Q)=h \cdot f_{1 t *}(1 / Q) \\
& f_{*}=\frac{E b t^{4}}{l^{2}} \cdot f_{2 h *}(Q)=\frac{E b h^{4}}{l^{2}} \cdot f_{2 t *}(1 / Q) \\
& \sigma_{\text {max }}=\frac{E t^{2}}{l^{2}} \cdot f_{3 h}(Q)=\frac{E h^{2}}{l^{2}} \cdot f_{3 t}(1 / Q)
\end{aligned}
$$

where $d_{*}$ and $f_{*}$ represent the different positions and force points respectively, and $f_{1 h *}(Q), f_{1 t *}(1 / Q), f_{2 h *}(Q), f_{2 t *}(1 / Q), f_{3 h}(Q)$ and $f_{3 t}(1 / Q)$ can be concluded from the expressions of $d_{*}, f_{*}$, and $\sigma_{\max }$ respectively.

The two expressions for each characteristic serve for presenting the influence of variating $h$ and $t$ separately. The expressions with $f_{1 h *}(Q), f_{2 h *}(Q)$ and $f_{3 h}(Q)$ represent the influence of variating $h$ separately while the expressions with $f_{1 t *}(1 / Q), f_{2 t *}(1 / Q)$ and $f_{3 t}(1 / Q)$ represent the influence of variating $t$ separately $(1 / Q=t / h)$. Two plots are presented in the following for each 
characteristic in (3) in order to visualize the influence of variating $h$ and $t$ respectively.

\subsubsection{Positions}

The variations of $d_{t o p}, d_{b o t}$ and $d_{\text {end }}$ with respect to $Q$ are demonstrated in Figure 5. These curves visualize the influence of variating $h$ for constant values of $t$. The curves $d_{b o t}$ and $d_{e n d}$ increase monotonically with respect to $Q$ while the curve $d_{t o p}$ decreases to a minimum at $Q=4.74$ and increases after that. All the three positions are directly proportional to $h$ for high and/or constant values of $\mathrm{Q}$.

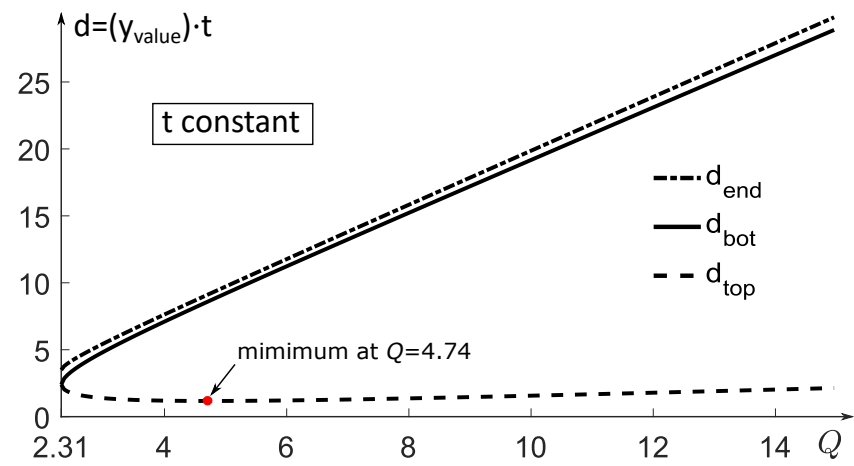

Figure 5: Curves of $d_{t o p}, d_{b o t}$ and $d_{\text {end }}$ with respect to $Q=h / t$. The curves show the influence of variating $h$ separately according to $f_{1 h *}$.

The variations of $d_{t o p}, d_{b o t}$ and $d_{\text {end }}$ with respect to $1 / Q=t / h$ are demonstrated in Figure 6. These curves visualize the influence of variating $t$ for constant values of $h$. It can be noticed that $d_{b o t}$ and $d_{e n d}$ decrease and $d_{t o p}$ increases with respect to $1 / Q$.

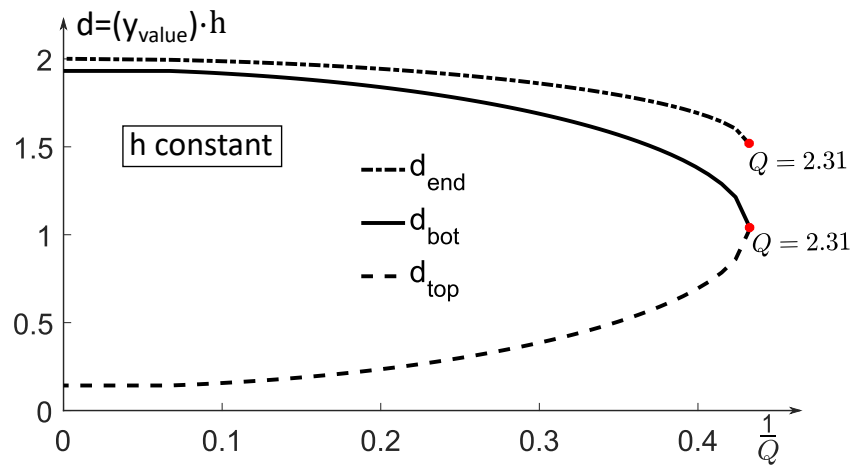

Figure 6: Curves of $d_{t o p}, d_{b o t}$ and $d_{\text {end }}$ with respect to $1 / Q=t / h$. The curves show the influence of variating $t$ separately according to $f_{1 t *}$. 
The values of the different snapping forces $f_{*}$ are proportional to the Young's modulus $E$, the depth $b$ and inversely proportional to the cubic value of the length $l^{3}$. The variations of $f_{\text {top }}$ and $f_{\text {bot }}$ with respect to $Q$ are demonstrated in Figure 7 .

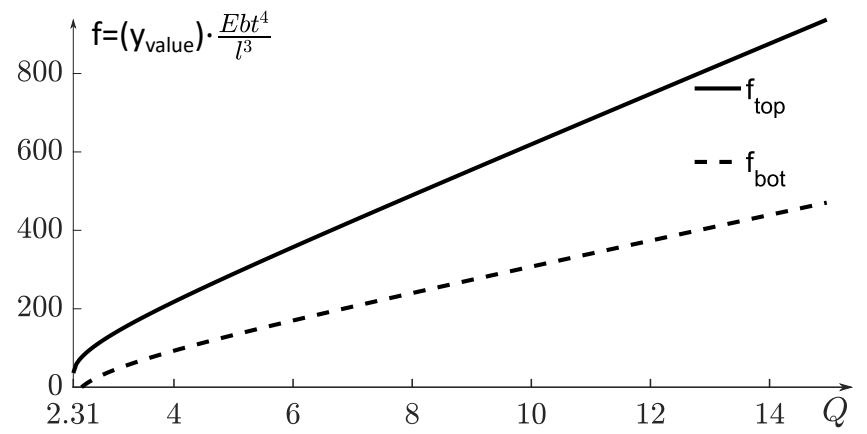

Figure 7: Curves of $f_{\text {top }}$ and $f_{\text {bot }}$ with respect to $Q=h / t$. The curves show the influence of variating $h$ separately according to $f_{2 h *}$.

Equation (3) and Figure 7 show that the snapping forces $f_{*}$ increases monotonically with respect to $h$ (according to $f_{2 h *}(Q)$ ) for constant values of the other parameters $(E, b, t$ and $l)$.

Analyzing the equations of $f_{\text {top }}$ and $f_{\text {bot }}$ in (1) shows that they have a maximum at $Q=2.52$ and $Q=3.00$ respectively when changing the value of the thickness $t$. The forces increase with $t$ after these values of $Q$ (lower values of $t / h)$, while they change inversely before that. The variations of $f_{\text {top }}$ and $f_{\text {bot }}$ with respect to $1 / Q$ are demonstrated in Figure 8.

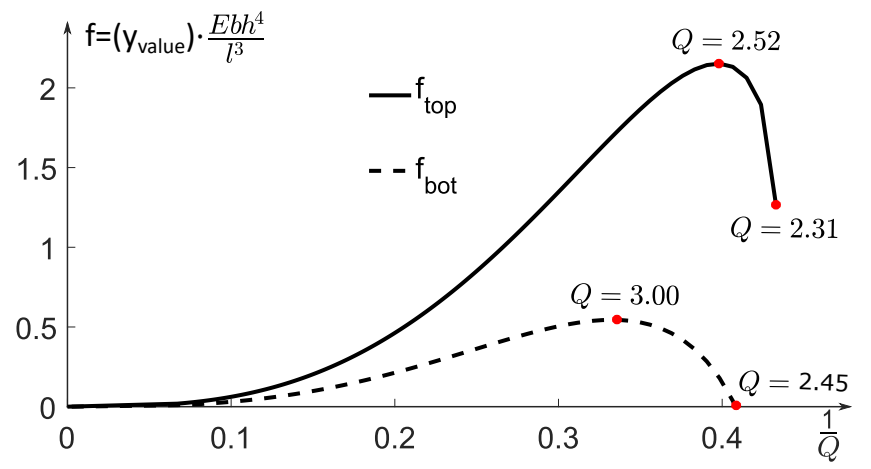

Figure 8: Curves of $f_{\text {top }}$ and $f_{\text {bot }}$ with respect to $1 / Q=t / h$. The curves show the influence of variating $t$ separately according to $f_{2 t *}$.

For constant values of $Q, f_{*}$ are proportional to the fourth power value of the thickness $t^{4}$ and/or the fourth power value of the height $h^{4}$. However, for 
high values of $Q, f_{*}$ are proportional to the cubic value of the thickness $t^{3}$ and to the height $h$.

\subsubsection{Maximal stress}

The value of the maximal stress $\sigma_{\max }$ is proportional to the Young's modulus $E$ and inversely proportional to the square value of the length $l^{2}$. The curves of

$\sigma_{\max }$ with respect to $Q$ (according to $f_{3 h}$ ) and with respect to $1 / Q$ (according to $f_{3 t}$ ) are demonstrated in Figures 9 and 10 respectively.

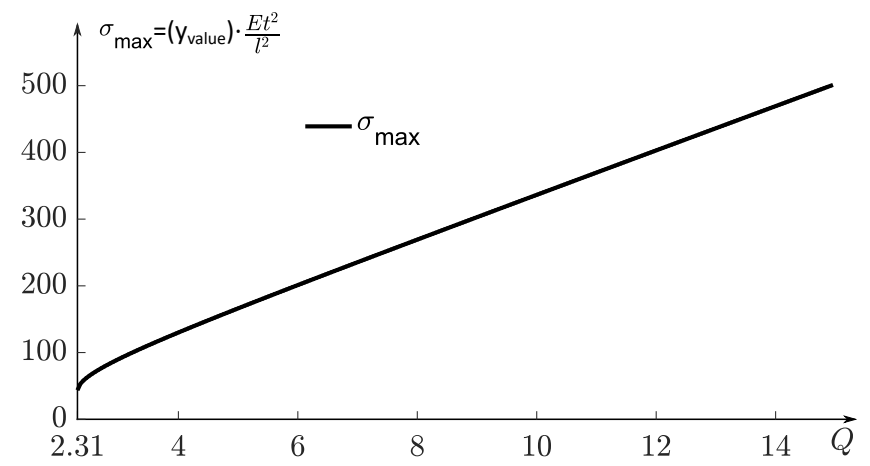

Figure 9: Curves of $\sigma_{\max }$ with respect to $Q=h / t$. The curves show the influence of variating $h$ separately according to $f_{3 h}$.

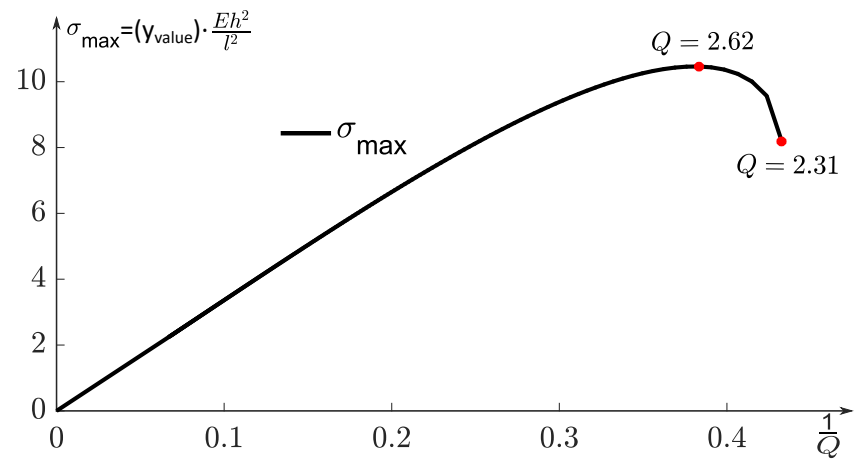

Figure 10: Curves of $\sigma_{\max }$ with respect to $1 / Q=t / h$. The curves show the influence of variating $t$ separately according to $f_{3 t}$.

As the case of the snapping forces, Figure 9 shows that $\sigma_{\max }$ increases monotonically with respect to the height $h$ for constant values of the other parameters $(E, t$ and $l)$.

$200 \quad$ Analyzing the equation of $\sigma_{\max }$ in (2) shows that the internal stress is maximized for $Q=2.62$ when variating the thickness $t$ for constant values of the height $h$. As demonstrated in Figure 10 the variations of $t$ and $\sigma_{\max }$ are proportional for $Q>2.62$, while they change inversely before that. 
For constant values of $Q, \sigma_{\max }$ is related proportionally to the square value values of $Q(Q>6), \sigma_{\max }$ is related proportionally to the thickness $t$ and the height $h$.

\subsection{Stress and miniaturization limits}

After defining the maximum value of the internal stress $\sigma_{\max }$, an important requirement in the design of a preshaped curved beam is that $\sigma_{\max }$ must remain under a critical limit $\sigma_{\text {crit }}$.

$$
\sigma_{\max }<\sigma_{\text {crit }}
$$

The critical limit $\sigma_{\text {crit }}$ can be determined according to the design preferences, it can be the yield strength, the fatigue limit, the fracture limit, etc..

Compliance with this criterion defines a critical limitation on the size of the curved beam, mainly in terms of miniaturization. The length $l$ is the main dimension in terms of size. Miniaturizing $l$ leads to higher values of the internal stresses and to reduce the security margin before reaching the stress limits. limits can be concluded from (4).

$$
l_{\text {min }}=\sqrt{\frac{E}{\sigma_{\text {crit }}}} \cdot t \cdot f_{4 h}(Q)=\sqrt{\frac{E}{\sigma_{\text {crit }}}} \cdot h \cdot f_{4 t}(1 / Q)
$$

where $f_{4 h}=\sqrt{ }\left(f_{3 h}\right)$ and $f_{4 t}=\sqrt{ }\left(f_{3 t}\right)$.

The minimal allowable length $l_{\text {min }}$ is higher when the material is more fragile $\left(\sigma_{\text {crit }} \searrow\right)$ and less flexible $(E \nearrow)$. As for the dimensions, $l_{\min }$ is proportional to $220 l \sqrt{\sigma_{\max }}$ as can be concluded from (2) and (4). Therefore, the variation of $l_{\min }$ with respect to $t, h$ and $Q$ is proportional to the square root of the variation of $\sigma_{\max }$ with respect to $t, h$ and $Q$. Idem, $l_{\min }$ is maximized for $Q=2.62$ when changing the value of the thickness $t$ for constant values of the other parameters $\left(E, \sigma_{\text {crit }}\right.$ and $\left.h\right)$.

225 The curves of $l_{\text {min }}$ with respect to $Q$ (according to $f_{4 h}$ ) and with respect to $1 / Q$ (according to $f_{4 h}$ ) are demonstrated in Figures 11 and 12 respectively.

Thereafter, the minimal possible length $l_{\text {min }}$ of a preshaped curved beam can be determined directly from (5). However, the most miniature size is not necessarily the optimal size that ensures all the design requirements. A design 230 methodology is presented in the next section that allows choosing the optimal dimensions and ensuring the design requirements of a preshaped curved beam.

\subsection{Summary of the parameters variation influence}

In light of the above, a variable scale influence is remarked between the material and dimension parameters and the different characteristics of the preshaped curved beam. Table 1 summarizes the effects of changing these parameters on the different characteristics $d_{*}, f_{*}, \sigma_{\max }$ and $l_{\min }$.

The arrow $\nearrow$ means that the concerned characteristic in the column increases when increasing the variable in the row. The arrow $\searrow$ means that it evolves in 


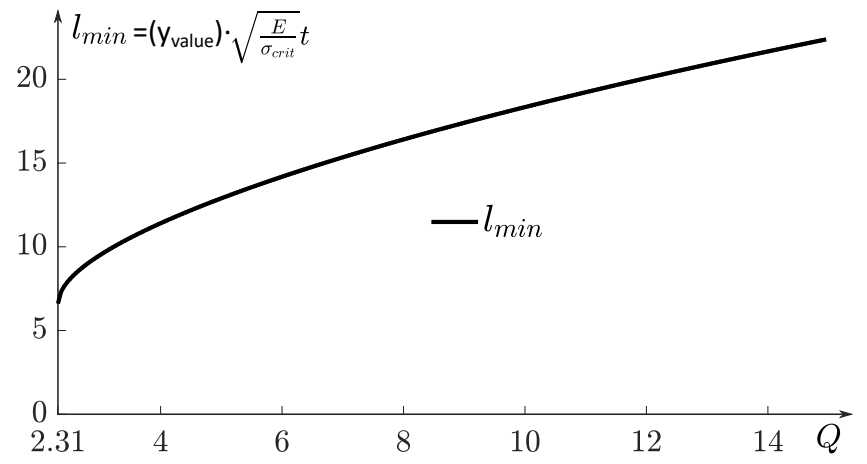

Figure 11: Curve of $l_{\text {min }}$ with respect to $Q=h / t$. The curve shows the influence of variating $h$ separately according to $f_{4 h}$.

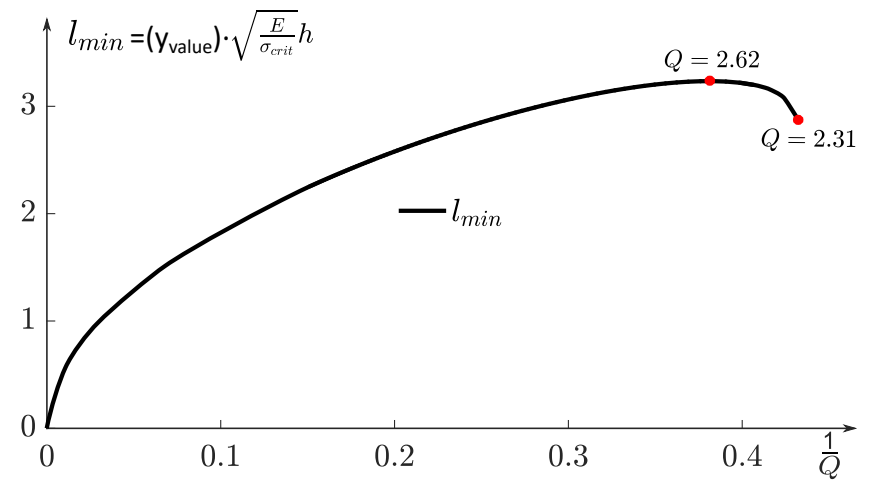

Figure 12: Curve of $l_{\min }$ with respect to $1 / Q=t / h$. The curve shows the influence of variating $t$ separately according to $f_{4 t}$.

the reverse direction. The power index means that the concerned characteristic evolves proportionally to the index power $(1 / 2,1,2,3,4)$ of the variable.

The parameter " $Q$ const." refers to the variation of $h$ and $t$ simultaneously with a constant ratio $Q$. The parameter "area" refers to the variation of $h, t$ and $l$ proportionally. This parameter clarifies the influence of scaling of the area of preshaped curved beams. In microfabrication, the device area determines the

245 number of devices per wafer. Thereby, the unit cost is related to the area of the device. The parameter "volume" refers to the variation of $b, h, t$ and $l$ proportionally. Besides, the depth of the wafer (or the device layer) is usually equivalent to the value of $b$. Therefore, knowing the influence of variating the volume is an economic indicator since it allows choosing the wafer and deter250 mining number of elements per wafer and the etching time which determine the fabrication cost. 
Table 1: Influence of the variation of the material and dimension parameters on the positions, snapping forces, maximal stress and limit of miniaturization of the length.

\begin{tabular}{|l|c|c|c|c|}
\hline Parameters & $d_{*}$ & $f_{*}$ & $\sigma_{\max }$ & $l_{\min }$ \\
\hline$E$ & - & $\nearrow^{1}$ & $\nearrow^{1}$ & $\nearrow^{\frac{1}{2}}$ \\
\hline$\sigma_{\text {crit }}$ & - & - & - & $\searrow^{\frac{1}{2}}$ \\
\hline$b$ & - & $\nearrow^{1}$ & - & - \\
\hline$l$ & - & $\nearrow^{3}$ & $\searrow^{2}$ & - \\
\hline$t$ & $\propto\left(f_{1 t *}\right)$ & $\propto\left(f_{2 t *}\right)$ & $\propto\left(f_{3 t}\right)$ & $\propto\left(f_{4 t}\right)$ \\
\hline$h$ & $\propto\left(f_{1 h *}\right)$ & $\nearrow\left(f_{2 h *}\right)$ & $\nearrow\left(f_{3 h}\right)$ & $\nearrow\left(f_{4 h}\right)$ \\
\hline$Q$ const. & $\nearrow^{1}$ & $\nearrow^{4}$ & $\nearrow^{2}$ & $\nearrow^{1}$ \\
\hline area & $\nearrow^{1}$ & $\nearrow^{1}$ & - & $\nearrow^{1}$ \\
\hline volume & $\nearrow^{1}$ & $\nearrow^{2}$ & - & $\nearrow^{1}$ \\
\hline
\end{tabular}

\section{Design optimization methodology}

In this section, a design optimization methodology of the preshaped curved beam is presented. The miniaturization of the curved beam is concerned in the design optimization while ensuring the required performance and a set of constraints. The performance of the curved beam bistable mechanism is characterized by the stroke $d_{s}$ between the two stable positions and the holding force $f_{h}$ at each stable position. The design constraints include a set of specifications that are defined according to a design requirement and some limitations that must be respected usually in any design.

After defining the different constraints, the design optimization problem is solved by a standard optimization algorithm (Sequential quadratic programming) and by visual interpretation. The results are exactly the same in the two cases which validates the calculation. However, the visual interpretation methodology presented in this paper involves the identification of the set of all the possible dimensions which satisfy the required performance and ensure the different constraints. This is not possible using the optimization algorithm which provides only the optimal dimensions.

A set of specifications is chosen to be considered in the design procedure in this paper. The choice of these specifications can be probably found in large number of design cases. The material and the depth $b$ are predefined (generally these variables are related to the choice of the wafers in microfabrication) and stop blocks are used to define the stable positions.

As for the stop blocks, other specifications are defined relatively. A sym275 metrical snapping force behavior between the two stable sides is concerned. Thereby, the stable positions are held with the same holding force value. In addition, the distance between the initial position and the first stop block must be higher than a defined distance. This last condition is related to some geo- 
metrical limits since minimal space is required for the practical fabrication and activation of stop blocks.

Besides, two main limitations are considered: a minimal achievable feature size called "fabrication limit" and a maximal allowable stress called "stress limit". The design methodology, limitations and specifications are summarized in the block diagram in Figure 13

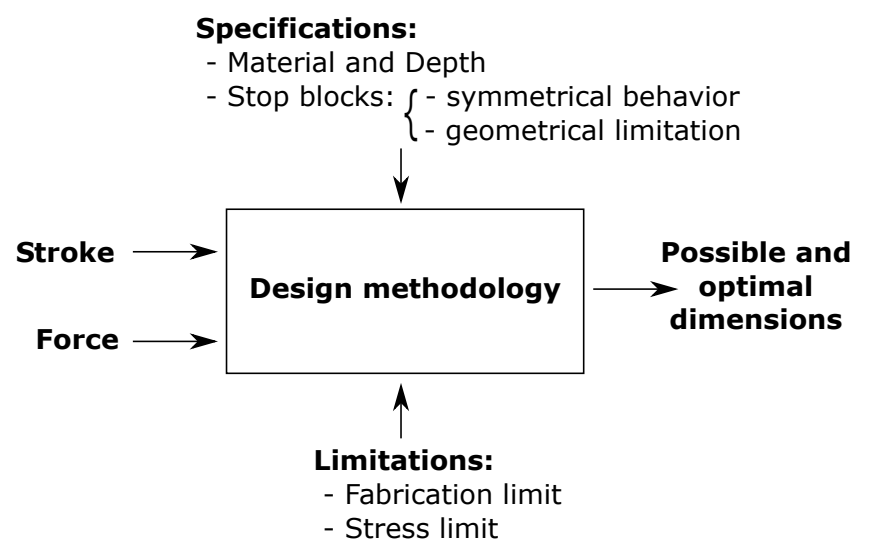

Figure 13: Block diagram representing the design methodology.

In the following, the different conditions on the dimensions are extracted. These conditions correspond to the required performance, specification and limitations. Afterwards, the optimization problem for a specific design is defined and solved. The field of possibilities for the dimensions reduces with each condition which leads in a final stage to define the miniature dimensions of the preshaped curved beam that allow reaching the desired performance with respecting the different constraints.

\subsection{Material}

Often, the material is chosen regarding the fabrication process or defined in the design specifications. The parameters that are related to the material are the Young's modulus $E$ and the stress limit $\sigma_{\text {crit }}$.

In terms of the stress, materials with lower $E / \sigma_{\text {crit }}$ ratio lead to more important margin of dimensions according to the stress limits. In terms of the snapping force, materials with higher values of $E$ generate higher forces.

\subsection{Stop blocks}

300

In the design specifications, stop blocks are used to define the stable positions. In this case, after fabrication, the curved beam is pushed manually beyond the first stop block. After that, the position of the curved beam is bounded between the two stop blocks at $d_{s 1}$ and $d_{s 2}$. Figure 14 shows the stable positions of the curved beam without and with the use of stop blocks. 


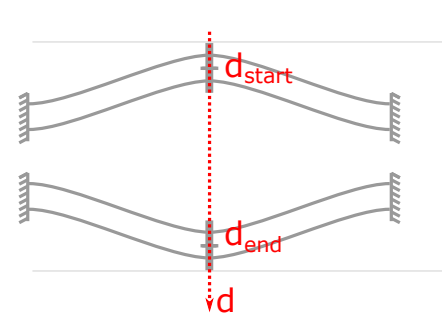

(a)

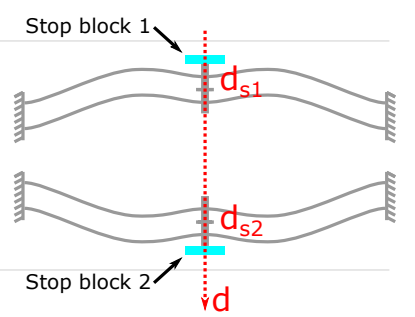

(b)

Figure 14: The two stable positions of the curved beam at $d_{\text {start }}$ and $d_{\text {end }}(\mathrm{a})$, the new stable positions at $d_{s 1}$ and $d_{s 2}$ which are imposed by two stop blocks (b). and defining the holding forces $f_{s 1}$ and $f_{s 2}$ which are the snapping forces at these positions. Figure 15 shows the main snapping points that are used in the design of a preshaped curved beam.

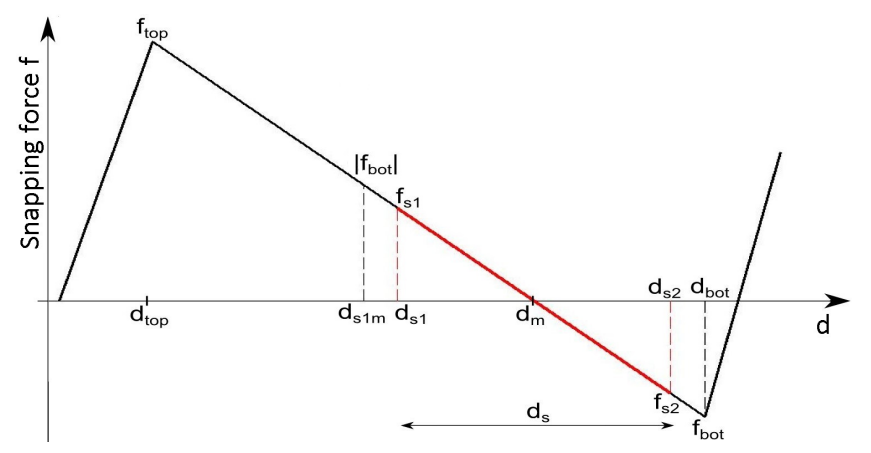

Figure 15: Important snapping points for the design of preshaped curved beams.

The stop blocks positions at $d_{s 1}$ and $d_{s 2}$ are symmetric between the positive and negative sides of the snapping forces (with respect to $d_{m}$ ).

$$
\left\{\begin{array}{l}
d_{s 1}=\frac{4}{3} h-\frac{d_{s}}{2} \\
d_{s 2}=\frac{4}{3} h+\frac{d_{s}}{2}
\end{array}\right.
$$

In this context, the value of the holding force $f_{h}$ at the stop blocks $\left(f_{h}=\right.$ $\left.f_{s 1}=f_{s 2}\right)$ is equivalent to:

$$
f_{h}=\frac{8 \pi^{2}}{3} \frac{E b d_{s} t^{3}}{l^{3}}
$$

Considering that the snapping forces must remain linear during deflection and symmetric with respect to $d_{m}$, the maximal stroke that can be defined is when the second stable position $d_{s 2}$ is at $d_{b o t}$. This is specially true since the 
distance between $d_{b o t}$ and $d_{m}$ is lower than the distance between $d_{t o p}$ and $d_{m}$.

The first stable position $d_{s 1}$ is at $d_{s 1 m}$ in this case. The maximal stroke $d_{s \max }$ $\left(d_{s \max }=d_{b o t}-d_{s 1 m}\right)$ is then expressed as follows:

$$
d_{\text {smax }}=-\frac{16}{27} h+\frac{4 \pi}{3} h \sqrt{\frac{1}{6}+\frac{16}{81 \pi^{2}}-\frac{1}{Q^{2}}}
$$

In light of the above, the stroke $d_{s}$ is limited by the value of $d_{s m a x}$. This leads to the following condition on $h$ and $t$ in order to ensure a linear behavior of the force along the stroke $d_{s}$.

$$
h \geq h_{d s}=\frac{2 d_{s}}{\pi^{2}}+\sqrt{\left(\frac{4}{\pi^{4}}+\frac{27}{8 \pi^{2}}\right) d_{s}^{2}+6 t^{2}}
$$

Another specification for the stop blocks is related to the first stop block position at $d_{s 1}$. After fabrication, the preshaped curved beam is pushed beyond the first stop block for activation. The first stop block is fabricated between the as-fabricated and the as-activated position of the preshaped curved beam. A minimal size of the first stop block in the design results in a minimal distance $d_{s 1 \text { min }}$ for the first stop block position. This leads to the following condition:

$$
d_{s 1} \geq d_{s 1 \min }
$$

Introducing the expression of $d_{s 1}$ in the last condition leads to a minimum on the value of $h$ independently from the other dimensions.

$$
h \geq h_{\text {min }}=\frac{3}{4} d_{s 1 \min }+\frac{3}{8} d_{s}
$$

\subsection{Limitations}

Two main limitations are considered in the design, the fabrication limit and the stress limit. A minimal feature size is generally allowed in the fabrication process. As the thickness is the smallest dimension, this leads to a condition on the thickness that must be equivalent or higher than the minimal feature size $t_{\min }$.

$$
t \geq t_{\text {min }}
$$

Concerning the stress limit, the maximal stress must remain lower than a critical limit (4). Besides, a minimum of the length $l$ can be determined from (7) to ensure the capacity of the curved beam to provide the holding force:

$$
l \geq l_{\text {min }}=t \sqrt[3]{\frac{8 \pi^{2}}{3} \frac{E b d_{s}}{f_{h}}}
$$

Comparing the value of $l_{\min }$ to provide $f_{h}$ in 13 with the value of $l_{\min }$ to respect the stress limits in (5), leads to the following condition on the height $h$ :

$$
h \leq h_{s}=\frac{t}{c_{1}}\left(c_{2}+\sqrt{\left(1+c_{1}\right) c_{2}^{2}+12 c_{1}}\right)
$$


with $c_{1}=1+\frac{64}{27 \pi^{2}}$ and $c_{2}=1-\sqrt[3]{\frac{3 b^{2} d_{s}^{2} \sigma_{\text {crit }}^{3}}{\pi^{2} E f f_{h}^{2}}}$.

Equation 14 shows that $Q=h / t$ must be lower than a determined value in order to ensure the symmetric holding forces without exceeding the stress limits. This value is related to the performance parameters $\left(d_{s}\right.$ and $\left.f_{h}\right)$, the material $\left(E\right.$ and $\left.\sigma_{\text {crit }}\right)$ and the depth $b$. Calling $\alpha$ this higher limit on $Q$, the value of $\alpha$ is equivalent to the following:

$$
\alpha=\frac{1}{c_{1}}\left(c_{2}+\sqrt{\left(1+c_{1}\right) c_{2}^{2}+12 c_{1}}\right)
$$

Later in Figure 16, $\alpha$ represents the slope of the curve line $h_{s}$ related to the

However, the depth $b$ must be higher than a minimal limit in order to provide the required performance in terms of the holding force and the stroke. Otherwise, for lower values of $b$, no possible dimensions allows the design requirements without exceeding the stress limits.

The holding force can be ensured by respecting the condition on $h$ which must be lower than $h_{s}$ as clarified in (14). Idem, the stroke can be ensured by respecting the condition on $h$ which must be higher than $h_{d s}$ as clarified in (9). Thereby, $h_{s}$ must be higher than $h_{d s}$ in order to ensure the existence of possible dimensions.

The curve of $h_{d s}$ with respect to thickness $t$ tends asymptotically towards a slope of $\sqrt{6}$ for high values of $t$. Hence, the slope $\alpha$ of $h_{s}$ must be higher than $\sqrt{6}$ in order to ensure the existence of possible dimensions that satisfy the 
different constraints and the required performance (see later Figure 16). This leads to the following condition on $b$ :

$$
b>b_{\min }=18.19 \frac{f_{h}}{d_{s} \sigma_{\text {crit }}} \sqrt{\frac{E}{\sigma_{\text {crit }}}}
$$

The minimal limit $b_{\text {min }}$ of $b$ is dependent only on the material properties

365 fabricate operational preshaped curved beams with required performance on a wafer with given material and dimensions.

Noting that for $b$ values very close to $b_{\min }$, high values of the other dimensions are required to ensure the required performance and constraints. If the consider the minimum of the other dimensions.

\subsection{Design optimization}

As the miniature dimensions of the preshaped curved beam are searched in this paper, the objective function in the design optimization is to minimize

375 the volume. The volume of the cube containing the curved beam in its initial position is considered in the optimization problem (volume $\left.=b l\left(h+\frac{t}{2}\right)\right)$. The optimization problem is summarized by the following:

$$
\text { subject to } \underset{b, t, h, l}{\operatorname{minimize}}\left\{\begin{aligned}
t \geq t_{\min } & \text { (fabrication limit) } \\
h h_{s} & \text { (stress limit) } \\
h \geq h_{\min } & \text { (geometrical constraint) } \\
h \geq h_{d s} & \text { (provide } d s) \\
l \geq l_{\min } & \text { (provide } \left.f_{h}\right) \\
b \text { constant } & \text { (wafer size) } \\
\text { material } & \text { (wafer material) }
\end{aligned}\right.
$$

The miniaturization problem in $(18)$ is solved by two methods. The first one is numerical using an optimization algorithm. The sqp (sequential quadratic programming) optimization algorithm available on MATLAB showed to be efficient to solve the optimization problem. The other method is by visual interpretation of the different conditions presented in (18). The visual interpretation method is detailed in the following.

Considering a predefined value of the depth $b$, it can be noticed that the condition on the length $\left(l_{\min }(13)\right)$ is proportional to the thickness $t$, while the other conditions on the thickness $t$ and the height $h\left(h_{d s}\left(\sqrt{9}, h_{\min }\right.\right.$ (11), $t_{\min }\left(12\right.$ ) and $h_{s}$ (14) ) are independent from the length $l$. This means that miniaturizing $t$ and $h$ separately leads necessarily to the miniaturization of $l$ and in results of the total volume which evolves monotonically with respect to $b, t, h$ and $l$.

The different conditions on $t$ and $h$ can be visualized in a $2 \mathrm{D}$ plot which shows clearly the range of possible dimensions for $t$ and $h$. The optimal dimensions in 
this range are the most miniature ones. Taking the following example, choosing a wafer with silicon material $\left(E=169 G P a, \sigma_{c r i t}=0.5 G P a\right)$ and a thickness $(b=$ $100 \mu \mathrm{m})$, considering minimal feature size $\left(t_{\min }=10 \mu \mathrm{m}\right)$ defining $d_{s 1 \mathrm{~min}}=45$ $\mu \mathrm{m}$ and setting the performance parameters $\left(d_{s}=30 \mu \mathrm{m}\right.$ and $\left.f_{h}=0.5 \mathrm{mN}\right)$. Figure 16 shows the field of possible dimensions of $t$ and $h$. The dimensions in the white part allow reaching the desired performance and respecting limitations and specifications.

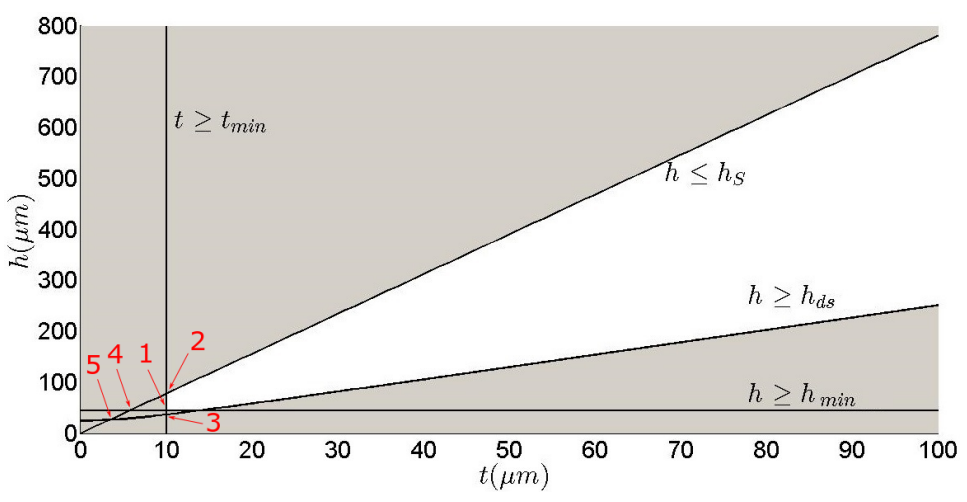

Figure 16: Field of possibilities for the thickness and the height. The possible dimensions are in the white part.

The points $1,2,3,4$, and 5 are the intersection between curves $\left(h_{\min }\right.$ and $\left.t_{\min }\right),\left(h_{s}\right.$ and $\left.t_{m i n}\right),\left(h_{d s}\right.$ and $\left.t_{m i n}\right),\left(h_{s}\right.$ and $\left.h_{m i n}\right)$ and $\left(h_{s}\right.$ and $\left.h_{d s}\right)$ respectively. The most miniature dimensions in Figure 16 correspond to point 1 $(t=10 \mu \mathrm{m}, h=45 \mu \mathrm{m})$. Thereby, the first stop block position is at $d_{s 1}=45 \mu \mathrm{m}$ and the second one is at $d_{s 2}=75 \mu \mathrm{m}$. The miniature length corresponding to point 1 is calculated from (13), it is equivalent to $l=2.988 \mathrm{~mm}$. In result, all the dimensions are obtained. Besides, it is noticed that these optimal dimensions are the same obtained with sqp optimization algorithm on Matlab. Noting that the optimization algorithm on Matlab converges to the same set of optimal dimensions. This may return to the convex nature of the problem where only one optimal solution (local and global) exists.

Nevertheless, variating the design inputs and constraints, the relative positions of the points 1-5 in Figure 16 can change. The points 1-5 may become inside or outside the zone of possible dimensions accordingly. Depending on the different cases clarified in Table 2, the resulting most miniature dimensions correspond to one of the points $1,3,4$ or $5 . h_{1}, \ldots, h_{5}$ denote the corresponding height of points $1, \ldots, 5$ respectively.

The different cases in Table 2 could be reached by variating $f_{h}$ and $d_{s}$. Figures 17, 18 and 19 show the optimal dimensions ( $t, h$, and $l$ respectively) obtained while variating $f_{h}$ and $d_{s}$.

420 Every point in the curves in Figures 17, 18 and 19 is a result of an optimization problem. The results calculated numerically with sqp optimization algorithm on Matlab and those calculated with an algorithm based on the visual 
Table 2: Different cases for the miniature dimensions of $t$ and $h$.

\begin{tabular}{|c|c|c|}
\hline & Cases & Optimal point \\
\hline Case 1 & $h 2<h 3 \& h 1<h 5$ & 5 \\
\hline Case 2 & $h 1<h 3<h 2$ & 3 \\
\hline Case 3 & $h 3<h 1<h 2$ & 1 \\
\hline Case 4 & $h 1>h 2 \& h 1>h 5$ & 4 \\
\hline Case 5 & $\alpha<\sqrt{6}$ & no solution \\
\hline
\end{tabular}

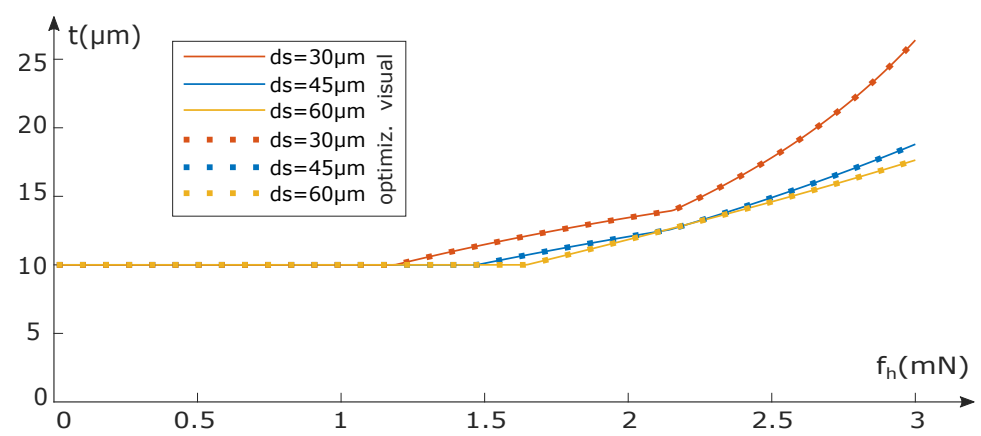

Figure 17: Optimal thickness dimension obtained by optimization algorithm and visual interpretation with variating $f_{h}$ and $d_{s}$.

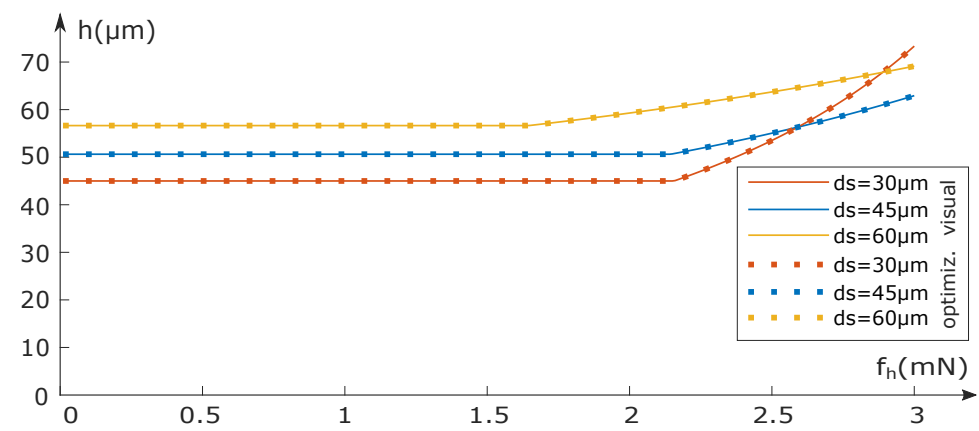

Figure 18: Optimal height dimension obtained by optimization algorithm and visual interpretation with variating $f_{h}$ and $d_{s}$.

methodology are exactly the same which validates the reasoning and calculation.

The curve $h_{d s}$ in Figure 16 is dependent on $d_{s}$ and independent from $f_{h}$.

${ }_{425}$ Three values of $d_{s}$ are considered while variating $f_{h}$ in the optimization results shown in Figures 17, 18 and 19. For $d_{s}=30 \mu \mathrm{m}$ and $d_{s}=45 \mu \mathrm{m}$, the curve $h_{d s}$ remains below point $1(h 3<h 1)$, while for higher values of $d_{s}\left(d_{s}=60 \mu m\right)$ the curve $h_{d s}$ becomes higher than point $1(h 3>h 1)$.

At low values of $f_{h}$, the curve $h_{s}$ is higher than point $1(h 2>h 1)$ and point ${ }_{430} 3(h 3>h 1)$. Thus the optimal dimensions are at point 1 for $d_{s}=30 \mu m$ and $d_{s}=45 \mu \mathrm{m}$, while it is at point 3 for $d_{s}=60 \mu \mathrm{m}$. 


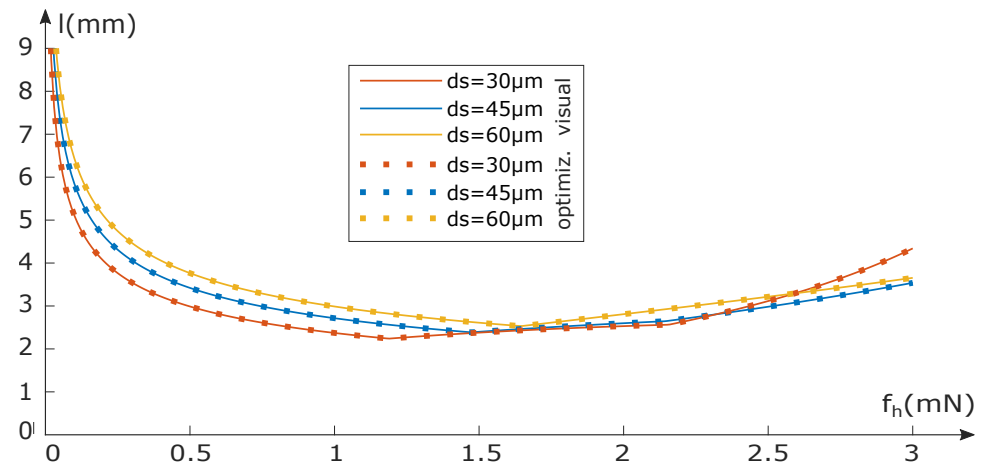

Figure 19: Optimal length dimension obtained by optimization algorithm and visual interpretation with variating $f_{h}$ and $d_{s}$.

While increasing $f_{h}$, the slope $\alpha$ of $h_{s}$ decreases and the curve $h_{s}$ becomes below point $1(h 2<h 1)$ and point $3(h 3<h 1)$ at certain limits. For $d_{s}=30 \mu m$ and $d_{s}=45 \mu \mathrm{m}$, at higher values of $f_{h}$, the optimal point becomes at point 4 and afterwards at point 5 . For $d_{s}=60 \mu m$, the optimal point becomes at point 5 for high values of $f_{h}$. The transition between the different cases of optimal points can be noticed in the change of slope of curves in Figures 17, 18 and 19 .

Furthermore, for higher values of $f_{h}$, the slope $\alpha$ of $h_{s}$ becomes closer to $\sqrt{6}$ and the different dimensions become extensively high. When $\alpha$ is below $\sqrt{6}$, no possible dimensions can satisfy the required performance and constraints. Normally, higher values of the depth $b$ must be considered in these cases in order to increase $\alpha$ and decrease the different dimensions. If so, it is preferable to choose the depth $b$ in a way that the curve $h_{s}$ is close to point 1 or point 3 (depending on the cases, $h_{3}>h_{1}$ or $h_{3}<h_{1}$ ). In this way, the length which 445 is the main dimension in terms of miniaturization can reach its most miniature possible value as shown in Figure 19

The design methodology presented in this paper is proposed for a design with some specific constraints. These constraints may vary from one design to another. The same methodology can be adopted for other designs with other specifications, where the corresponding conditions must be extracted firstly and the range of possible dimensions are defined subsequently. The design methodology can be changed to suit each design requirement based on the design elements provided in this paper.

\section{Conclusion}

The design of a preshaped curved beam used as a bistable mechanism in MEMS applications was investigated in this paper. After introducing the model, the influence of variating the different parameters (material properties and dimensions) on the behavior of the preshaped curved beam was analyzed. The limit of miniaturization of the beam related to the stress limit was also identified. A design optimization methodology was then proposed. After defining the 
different constraints, the miniature dimensions are searched either numerically using an optimization algorithm or either by visual interpretation methodology. This methodology allows identifying the field of possible dimensions that allows a desired performance and respecting the design specifications and limitations. This is possible thanks to the explicit modeling expressions obtained from the modeling with high modes of buckling, which showed high accordance with FEM simulations and experiments. Afterwards, the most miniature dimensions are identified among the field of possible dimensions. The results in different cases of optimization problems showed to be the same obtained using the optimization

470 algorithm and the proposed methodology which validates the reasoning and the calculation.

\section{References}

[1] S. S. H. Zaidi, Contactless energy transfer and control strategy for bistable micro-actuator, Ph.D. thesis, Université de Technologie de Compiègne (2011).

[2] B. Camescasse, Actionnements statique et dynamique d'un mécanisme bistable: aspects modélisation, conception et expérimental, Ph.D. thesis, Université Pierre et Marie Curie (2013).

[3] P. Cazottes, Actionnement des systèmes bistables : modélisation et études expérimentales, Ph.d. thesis, Université Pierre et Marie Curie, Paris, France (2009).

[4] I. Z. Pane, T. Asano, Investigation on bistability and fabrication of bistable prestressed curved beam 47 (2008) 5291.

[5] B. Charlot, W. Sun, K. Yamashita, H. Fujita, H. Toshiyoshi, In-plane bistable nanowire for memory devices, in: Design, Test, Integration and Packaging of MEMS/MOEMS, 2008. MEMS/MOEMS 2008. Symposium on, IEEE, 2008, pp. 254-258.

[6] B.-T. Liao, H.-H. Shen, H.-H. Liao, Y.-J. Yang, A bi-stable 2x2 optical switch monolithically integrated with variable optical attenuators, Optics express 17 (22) (2009) 19919-19925.

[7] S. Park, D. Hah, Pre-shaped buckled-beam actuators: theory and experiments, Sensors and Actuators A: Physical 148 (1) (2008) 186-192.

[8] J. Qiu, J. Lang, A. Slocum, A curved-beam bistable mechanism 13 (2) (2004) 137146. doi:10.1109/JMEMS. 2004.825308.

495 [9] H. Hussein, P. Le Moal, G. Bourbon, Y. Haddab, P. Lutz, Modeling and stress analysis of a pre-shaped curved beam: Influence of high modes of buckling, International Journal of Applied Mechanics 7 (04) (2015) 1550055 . 
[10] S. Krylov, B. R. Ilic, S. Lulinsky, Bistability of curved microbeams actuated by fringing electrostatic fields, Nonlinear Dynamics 66 (3) (2011) 403.

[11] F. Tajaddodianfar, H. N. Pishkenari, M. R. H. Yazdi, E. M. Miandoab, Size-dependent bistability of an electrostatically actuated arch nems based on strain gradient theory, Journal of Physics D: Applied Physics 48 (24) (2015) 245503.

[12] M. D. Williams, F. van Keulen, M. Sheplak, Modeling of initially curved beam structures for design of multistable mems, Journal of Applied Mechanics 79 (1) (2012) 011006.

[13] S. A. Emam, A. H. Nayfeh, On the nonlinear dynamics of a buckled beam subjected to a primary-resonance excitation 35 (1) (2004) 117. doi:10. 1023/B:NODY.0000017466.71383.d5

[14] A. H. Nayfeh, S. A. Emam, Exact solution and stability of postbuckling configurations of beams 54 (4) (2008) 395408. doi:10.1007/ s11071-008-9338-2.

[15] F. Tajaddodianfar, M. R. H. Yazdi, H. N. Pishkenari, Nonlinear dynamics of mems/nems resonators: analytical solution by the homotopy analysis method, Microsystem Technologies (2016) 1-14.

[16] Y.-J. Yang, B.-T. Liao, W.-C. Kuo, A novel $2 \times 2$ mems optical switch using the split cross-bar design, Journal of Micromechanics and Microengineering 17 (5) (2007) 875.

${ }_{520}$ [17] H. Hussein, V. Chalvet, P. Le Moal, G. Bourbon, Y. Haddab, P. Lutz, Design optimization of bistable modules electrothermally actuated for digital microrobotics, in: Advanced Intelligent Mechatronics (AIM), 2014 IEEE/ASME International Conference on, IEEE, 2014, pp. 1273-1278.

[18] J. Qiu, J. H. Lang, A. H. Slocum, A. C. Weber, A bulk-micromachined bistable relay with u-shaped thermal actuators, Microelectromechanical Systems, Journal of 14 (5) (2005) 1099-1109.

[19] R. Vitushinsky, S. Schmitz, A. Ludwig, Bistable thin-film shape memory actuators for applications in tactile displays, Microelectromechanical Systems, Journal of 18 (1) (2009) 186-194.

[20] F. Tajaddodianfar, H. N. Pishkenari, M. R. H. Yazdi, E. M. Miandoab, On the dynamics of bistable micro/nano resonators: analytical solution and nonlinear behavior, Communications in Nonlinear Science and Numerical Simulation 20 (3) (2015) 1078-1089.

[21] H. Farokhi, M. H. Ghayesh, S. Hussain, Pull-in characteristics of electrically actuated mems arches, Mechanism and Machine Theory 98 (2016) 133-150. 
[22] H. Hussein, Contribution to digital microrobotics: modeling, design and fabrication of curved beams, u-shaped actuators and multistable microrobots, Ph.D. thesis, Université Bourgogne Franche-Comté (2015).

[23] M. Vangbo, An analytical analysis of a compressed bistable buckled beam 69 (3) (1998) 212216. doi:10.1016/S0924-4247(98)00097-1. 